This is the peer reviewed version of the following article: R. Ruiz-González, R. Bresoli-Obach, Ò. Gulías, M. Agut, H. Savoie, R. W. Boyle, S. Nonell, F. Giuntini, Angew. Chem. Int. Ed. 2017, 56, 2885, which has been published in final form at http://dx.doi.org/10.1002/ anie.201609050. This article may be used for non-commercial purposes in accordance With Wiley Terms and Conditions for selfarchiving.

1

2

3

4

5

6

\title{
NanoSOSG: a Nanostructured Fluorescent Probe for Intracellular Singlet Oxygen Detection
}

\author{
Rubén Ruiz-González, ${ }^{[[a]}$ Roger Bresolí-Obach, ${ }^{[a]}$ Òscar Gulías, ${ }^{[a]}$ Montserrat Agut, ${ }^{[a]}$ Huguette \\ Savoie, ${ }^{[b]}$ Ross W. Boyle, ${ }^{*[b]}$ Santi Nonell ${ }^{*[a]}$ and Francesca Giuntini ${ }^{*[b, c]}$ \\ This paper is dedicated to the memory of Professor Giulio Jori, mentor and friend
}

\begin{abstract}
A biocompatible fluorescent nanoprobe for singlet oxygen $\left({ }^{1} \mathrm{O}_{2}\right)$ detection in biological systems has been designed, synthesised and characterized, that circumvents many of the limitations of the molecular probe Singlet Oxygen Sensor Green ${ }^{\circledR}$ (SOSG). This widely used commercial singlet oxygen probe has been covalently linked to a polyacrylamide nanoparticle core using different architectures to optimize the response to ${ }^{1} \mathrm{O}_{2}$. In contrast with its molecular counterpart, the optimum SOSG-based nanoprobe, which we call NanoSOSG, is readily internalised by $E$. coli cells, does not interact with BSA, does not change its spectrum inside cells and responds to intracellularly-generated ${ }^{1} \mathrm{O}_{2}$ by increasing its fluorescence.
\end{abstract}

Singlet oxygen $\left({ }^{1} \mathrm{O}_{2}\right)$ is the lowest excited electronic state of molecular oxygen and is endowed with atypical but appealing physico-chemical properties and behavior that puts it at the forefront of research in many different disciplines. ${ }^{[1]}$ Great efforts have been made in developing techniques and/or methods able not only to detect, but also to quantify the generation of ${ }^{1} \mathrm{O}_{2}$. Chemical probes react with ${ }^{1} \mathrm{O}_{2}$ leading to changes of a measurable physical property such as absorption, fluorescence, chemiluminescence or spin signal. ${ }^{[2]}$ Most ${ }^{1} \mathrm{O}_{2}$ probes are highly hydrophobic and, thus, lack sufficient water solubility and cell permeability. Although water-soluble chemical probes exist, ${ }^{[3]}$ they often fail to produce reliable results inside cells ${ }^{[4]}$ or present marked synthetic complexity. ${ }^{[5]}$ On the other hand, a large fraction of the ${ }^{1} \mathrm{O}_{2}$ produced inside a cell is rapidly quenched, resulting in a very short lifetime ${ }^{[6]}$ and hence reducing the probability of it being captured by the probe.

There is an emerging trend of using nanoparticles (NPs) for drug delivery, ${ }^{[7]}$ sensing ${ }^{[8]}$ and imaging. ${ }^{[9]}$ However, only a few reports exist on the use of ${ }^{1} \mathrm{O}_{2}$ traps associated with NPs. Among the emerging nanomaterials for biomedical applications, polyacrylamide is attracting much interest owing to its biocompatibility and chemical versatility. Successful examples of its use have recently been published in several fields. ${ }^{[10]}$ Synthesis of polyacrylamide NPs is straightforward and it allows tailoring both the size and the functionalization of the

[a] R. Ruiz-González, R. Bresolí-Obach, Ò. Gulías, M. Agut, S. Nonell Institut Químic de Sarrià, Universitat Ramón Llull,

Via Augusta 390, E-08019, Barcelona, Spain

E-mail: santi.nonell@iqs.url.edu

[b] H. Savoie, R.W. Boyle, F. Giuntini Department of Chemistry

University of Hull

Cottingham Road, Kingston upon Hull, HU6 7RX, United Kingdom

E-mail: R.W.Boyle@hull.ac.uk

[c] F. Giuntini

School of Pharmacy and Biomolecular Sciences

Liverpool John Moores University

Liverpool, L3 3AF, United Kingdom

E-mail: F.Giuntini@ljmu.ac.uk

These authors contributed equally to this work

Supporting information for this article is given via a link at the end of the document. nanospecies to suit the needs of specific applications. The porosity of polyacrylamide allows analytes (e.g., ions, small molecules, etc.) to diffuse within the NP and interact with the sensing moiety making it an ideal support for nanosensors. ${ }^{[11]}$ Numerous examples of biosensors based on polyacrylamide NPs are reported in the literature. ${ }^{[12]}$ In this work we present results on the synthesis, photochemical behavior and performance of polyacrylamide-based ${ }^{1} \mathrm{O}_{2}$-fluorescent nanoprobes

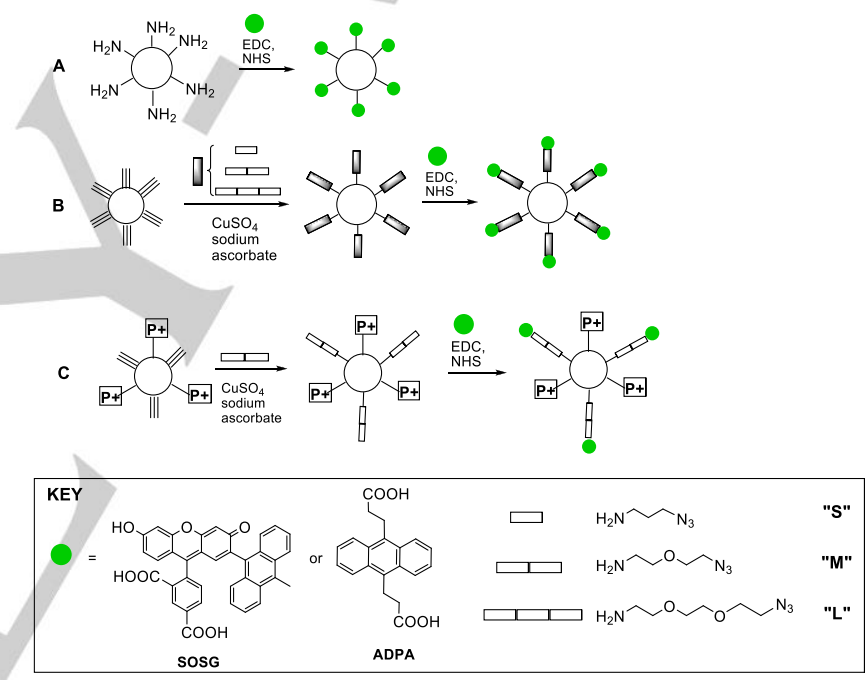

Scheme 1. Conjugation of ADPA or SOSG to functionalised polyacrylamide NPs, in the absence (A) and in the presence (B) of a spacer, and with positively-charged trimethylphosphonium groups $(\mathrm{C})$.

As an initial approach we developed a sensor in which ${ }^{1} \mathrm{O}_{2}$ chemical traps were directly attached to the NP, as depicted in Scheme 1, panel A. Amino-derivatised NPs were covalently bound to Singlet Oxygen Sensor Green ${ }^{\circledR}$ (SOSG) ${ }^{[3 f]}$ through its most reactive carboxylic group. ${ }^{[4 b]}$ SOSG is an anthracenefluorescein dyad in which the fluorescence is quenched by photoinduced intramolecular electron between the two moieties. Upon anthracene endoperoxidation in the presence of ${ }^{1} \mathrm{O}_{2}$, the electron transfer is blocked restoring the fluorescein's intrinsic fluorescence. ${ }^{[4 b]}$ According to the data supplied by the manufacturer, SOSG is a probe with a high specificity towards singlet oxygen ${ }^{[13]}$ and there is no chemical rational for expecting alteration of its specificity in the nanoparticle, because we link it via formation of an amide bond at a site that is rather remote from the reactive anthracene moiety. ${ }^{[14]}$ Aqueous solutions containing $2 \mathrm{mg} / \mathrm{mL}$ of these nanoprobes and $1 \mu \mathrm{M}$ of new methylene blue (NMB) as ${ }^{1} \mathrm{O}_{2}$ source were irradiated and the probe fluorescence changes observed over time. The response 
of the nanoprobe was poor when compared to the free probe, i.e., only a modest $10 \%$ fluorescence increase was observed (Figure $S 1$, panels $B$ and $D$ ). Even in deuterium oxide $\left(D_{2} O\right)$, where the 20 -fold longer ${ }^{1} \mathrm{O}_{2}$ lifetime facilitates the reaction, ${ }^{[1]}$ the fluorescence enhancement was below $40 \%$. In order to discard any effects of light scattering by the NPs, molecular SOSG solutions were irradiated in the presence of $1 \mu \mathrm{M} \mathrm{NMB}$ and increasing amounts of NPs (Figure S2). Our experiment shows that for concentrations up to $5 \mathrm{mg} / \mathrm{mL}$ SOSG behaves almost equally with or without added free NPs.

The poor performance of this early nanoprobe was ascribed to nonspecific interactions occurring between the probe and the polyacrylamide matrix, leading to decreased reactivity of the SOSG molecule bound to the polymer network. In addition, it is worth noting that the initial fluorescence of SOSG-NPs was substantially higher than that of free SOSG, which suggests that the SOSG microenvironment in the nanoprobe impairs efficient electron-transfer quenching.

Thus, spacers of different lengths were introduced to separate the polyacrylamide scaffold and the probe. To this end, alkyne-functionalised polyacrylamide particles were prepared, to allow orthogonal copper-catalysed azide-alkyne cycloaddition (CuAAC) coupling of the amino-azide functionalised linkers. ${ }^{[13][15]}$ Following CuAAC, the SOSG probe was attached to the free end of the linker via amide bond formation. (Scheme 1, panel B) Linkers of different size were tested (S, M, and L). Inclusion of linkers improved the performance of the nanoprobes. The best ${ }^{1} \mathrm{O}_{2}$ trapping efficiency was observed for the nanoprobe with the medium-size linker ( $M, 7.1 \AA$ ), which showed a fluorescence enhancement of up to 3.2 -fold in $\mathrm{D}_{2} \mathrm{O}$ after 50 min irradiation. This nanoprobe (henceforth NanoSOSG) was selected for further experiments. Its surface was further functionalized with cationic groups to facilitate cell uptake.

SOSG is described as cell-impermeant, ${ }^{[14][15]}$ which detracts from its usefulness as ${ }^{1} \mathrm{O}_{2}$ probe in biological media. Gollmer et al. concluded that this was due extensive protein binding in the culture medium. Indeed the presence of bovine serum albumin (BSA) resulted in red-shifted fluorescence and lower responsiveness to ${ }^{1} \mathrm{O}_{2}$, which the authors attributed to the protein kinetically competing with SOSG for ${ }^{1} \mathrm{O}_{2}$ molecules. Moreover, they were able to show that SOSG could in fact be internalised by HeLa cells in protein-free medium. ${ }^{[4 b]}$ Yet, intracellular SOSG showed additional problems that further detract from its use as a ${ }^{1} \mathrm{O}_{2}$ reporter: its fluorescence spectrum was still red-shifted compared to aqueous solutions, intense fluorescence was still observed prior to ${ }^{1} \mathrm{O}_{2}$ exposure, and it was difficult to obtain systematic and reproducible results. ${ }^{[4 b]}$ As shown below, the performance of NanoSOSG probes is not affected by such shortcomings.

In a first instance, the interaction with proteins, which heavily affects the fluorescence of SOSG, ${ }^{[15][16]}$ causes only a marginal fluorescence quenching of NanoSOSG at the highest BSA concentration (Figure S3), indicating that the interactions of the nanoprobe with the protein are prevented by the NP scaffold. We show in the Supplementary Information that SOSG actually forms $1: 1$ and 2:1 complexes with BSA, each with distinct spectra and binding constants (Figure S4). Similar situations are likely to occur in protein-rich environments as in cells. This evidence suggests NanoSOSG to be more suited than molecular SOSG as a fluorescent probe for ${ }^{1} \mathrm{O}_{2}$ in biological media.

The ability to NanoSOSG to respond to intracellular ${ }^{1} \mathrm{O}_{2}$ was then assessed through a series of assays using wild type (wt) $E$. coli and a genetically-modified $E$. coli strain that expresses miniSOG, a flavin-binding fluorescent protein with strong capacity to sensitise ${ }^{1} \mathrm{O}_{2}$ production inside cells. ${ }^{[16][17]}$ Figure 1 presents the behaviour of the free SOSG probe in both types of cells.

Figure $1 \mathrm{~A}$ shows that the fluorescence spectrum of SOSG in the presence of wt $E$. coli cells matches that in PBS, indicating that it is not bound to proteins. The effects of irradiation at $420 \pm 20 \mathrm{~nm}$ (see Figure S5 for details) are shown in Figures 1B and 1C. In wt cells the fluorescence of SOSG increases linearly as a result of its well-known self-sensitised photooxidation. ${ }^{[3 d]}$ In miniSOGexpressing cells, the rate of fluorescence increase shows two distinct regions: up to $15 \mathrm{~min}$ irradiation, the fluorescence increases at a rate similar to that observed in wt cells, whereas after that the rate increases approximately by 2.5 -fold. The viability of wt cells is not compromised by irradiation of SOSG whereas cells expressing miniSOG are killed very effectively, with more than $90 \%$ being killed after just 15 min (Figure 1C). Taken together, these results indicate that SOSG and miniSOG are not in close proximity at the early stages of irradiation, i.e., SOSG is not internalized by $E$. coli cells. The fluorescence increase observed in both cells at these early times is due solely to SOSG self-sensitised ${ }^{1} \mathrm{O}_{2}$ produced in the outer aqueous media and it is therefore independent of intracellular ${ }^{1} \mathrm{O}_{2}$. In miniSOG-expressing cells, intracellular ${ }^{1} \mathrm{O}_{2}$ damages the cell from the inside ${ }^{[17][18]}$ and eventually miniSOG is released to the external medium where it enhances the rate of SOSG photooxidation. An alternative explanation such as photochemical internalization of SOSG ${ }^{[18][19]}$ can be ruled out because no spectral changes are observed after irradiation (Figure 1A).

The corresponding results for NanoSOSG are also shown in Figure 1. As expected, NanoSOSG does not interact with cell proteins neither before nor after extensive irradiation (Figure 1B).The rate of fluorescence increase in wt and miniSOGexpressing cells is different already at early irradiation stages (Figure 1E), indicating that NanoSOSG is close to miniSOG from the onset, i.e., NanoSOSG has been internalised by E. coli. This is confirmed by the results of cell photoinactivation studies, which show cell mortality in wt bacteria (Figure 1F) due to intracellular ${ }^{1} \mathrm{O}_{2}$ photosensitised by NanoSOSG. In miniSOGexpressing cells, both a higher fluorescence increase and an enhanced cell photokilling can be observed, consistent with a higher rate of intracellular ${ }^{1} \mathrm{O}_{2}$ production due to miniSOG. Crucially, NanoSOSG is devoid of any measurable dark toxicity (Figure 1F). NanoSOSG-sensitized photoinactivation of bacteria should be of lesser importance in imaging experiments because the light doses used are typically at least one order of magnitude lower than those used in this paper. ${ }^{[20]}$ Moreover, it can be totally avoided in photosensitisation experiments by using an excitation wavelength where SOSG does not absorb. 

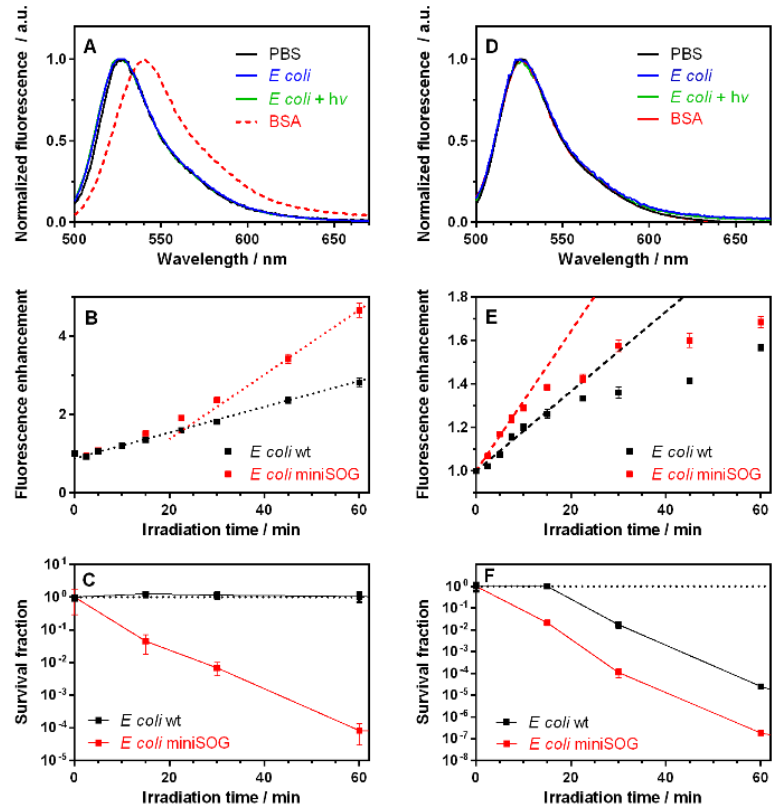

Figure 1. Comparison of SOSG (left panels) and NanoSOSG (right panels). (A) Fluorescence spectra of SOSG in PBS, in E. coli before and after irradiation, and in the presence of $200 \mu \mathrm{M}$ BSA. (B) Fluorescence enhancement of SOSG in wt (black) and miniSOG-expressing (red) E. coli cells as a function of the irradiation time $\left(\lambda_{\operatorname{exc}}=420 \pm 20 \mathrm{~nm}\right)$. (C) Photoinactivation of wt (black) and miniSOG-expressing (red) E. coli cells preincubated with SOSG as a function of the irradiation time $\left(\lambda_{\mathrm{exc}}=420 \pm 20 \mathrm{~nm}\right.$ ). (D) Fluorescence spectra of NanoSOSG in PBS, in E. coli before and after irradiation, and in the presence of $200 \mu \mathrm{M}$ BSA. (E) Fluorescence enhancement of NanoSOSG in wt (black) and miniSOG-expressing (red) E. coli cells as a function of the irradiation time $\left(\lambda_{\mathrm{exc}}=420 \pm 20 \mathrm{~nm}\right)$. (F) Photoinactivation of wt (black) and miniSOG-expressing (red) E. coli cells preincubated with SOSG as a function of the irradiation time.

In summary, we have designed, synthesised and characterised a polyacrylamide-based biocompatible fluorescent nanoprobe for ${ }^{1} \mathrm{O}_{2}$ detection in biological systems that circumvents many of the limitations of the widely used molecular probe SOSG. Thus, NanoSOSG is successfully internalised by E. coli cells without appreciable dark toxicity and correctly responds to intracellularly generated ${ }^{1} \mathrm{O}_{2}$. The "nano" approach has proved useful to extend the use of an existing and valuable fluorescent probe to complex biological systems.

\section{Acknowledgements}

Financial support for this research was obtained from the Spanish Ministerio de Economía y Competitividad (CTQ201020870-C03-01 and CTQ2013-48767-C3-1-R). R.B-O thank the Generalitat de Catalunya (DURSI) and the European Social Fund for predoctoral fellowships (2015 FI_B 00315). R.R-G thanks the Spanish Ministerio de Educación Cultura y Deporte for a "Beca de movilidad para estudiantes en programas de Doctorado con Mención hacia la excelencia" fellowship. OG thanks IQS for his predoctoral fellowship. FG and RWB thank EPSRC for funding (EP/H000151/1).

Keywords: fluorescence $\cdot$ intracellular $\cdot$ nanosensors $\cdot$ optical sensors $\cdot$ singlet oxygen

[1] a) C. Schweitzer, R. Schmidt, Chem. Rev. 2003, 103, 1685-1758; b) P. R. Ogilby, Chem. Soc. Rev. 2010, 39, 3181-3209; O. Planas, E. BoixGarriga, B. Rodríguez-Amigo, J. Torra, R. Bresolí-Obach, C. Flors, C. Viappiani, M. Agut, R. Ruiz-González, S. Nonell, in Photochemistry, Vol. 42 (Eds.: A. Albini and E. Fasani), The Royal Society of Chemistry, London, 2015, pp. 233-278.

[2] a) É. Hideg, in Methods Mol. Biol. (Ed.: R. Carpentier), Institute of Plant Biology, Biological Research Center, Szeged, 2004, pp. 249-260; b) B R. Rabello, A. P. Gerola, D. S. Pellosi, A. L. Tessaro, J. L. Aparício, W. Caetano, N. Hioka, J. Photochem. Photobiol. A Chem. 2012, 238, 5362; c) H. Wu, Q. Song, G. Ran, X. Lu, B. Xu, Trends Anal. Chem. 2011, 30, 133-141; d) G. Nardi, I. Manet, S. Monti, M. A. Miranda, V. Lhiaubet-Vallet, Free Radic. Biol. Med. 2014, 77, 64-70; e) R. RuizGonzález, A. L. Zanocco, in Singlet Oxygen: Applications in Biosciences and Nanosciences. Vol. 2 (Eds.: S. Nonell, C. Flors), The Royal Society of Chemistry, London, 2016, pp. 103-120.

[3] a) F. N. Castellano, J. R. Lakowicz, Photochem. Photobiol. 1998, 67, 179-183; b) N. Umezawa, K. Tanaka, Y. Urano, K. Kikuchi, T. Higuchi, T. Nagano, Angew. Chem. 1999, 38, 2899-2901; c) V. Nardello, J.-M. Aubry, P. Johnston, I. Bulduk, A. H. de Vries, P. L. Alsters, Synlett 2005, 2005, 2667-2669; d) X. Ragàs, A. Jiménez-Banzo, D. Sánchez-García, X. Batllori, S. Nonell, Chem. Commun. 2009, 20, 2920-2922; e) M. S. Oliveira, D. Severino, F. M. Prado, J. P. F. Angeli, F. D. Motta, M. S. Baptista, M. H. G. Medeiros, P. Di Mascio, Photochem. Photobiol. Sci. 2011, 10, 1546-1555; f) C. Flors, M. J. Fryer, J. Waring, B. Reeder, U. Bechtold, P. M. Mullineaux, S. Nonell, M. T. Wilson, N. R. Baker, J. Exp. Bot. 2006, 57, 1725-1734.

[4] a) B. Song, G. Wang, M. Tan, J. Yuan, J. Am. Chem. Soc. 2006, 128, 13442-13450; b) A. Gollmer, J. Arnbjerg, F. H. Blaikie, B. W. Pedersen, T. Breitenbach, K. Daasbjerg, M. Glasius, P. R. Ogilby, Photochem Photobiol. 2011, 87, 671-679; c) D. Kessel, M. Price, Photochem. Photobiol. 2012, 88, 717-720.

[5] D. Arian, L. Kovbasyuk, A. Mokhir, J. Am. Chem. Soc. 2011, 133 3972-3980.

[6] M. K. Kuimova, S. W. Botchway, A. W. Parker, M. Balaz, H. A. Collins H. L. Anderson, K. Suhling, P. R. Ogilby, Nat. Chem. 2009, 1, 69-73.

[7] a) D. Bechet, P. Couleaud, C. Frochot, M.-L. L. Viriot, F. Guillemin, M. Barberi-Heyob, Trends Biotechnol. 2008, 26, 612-621; b) K. Cho, X. Wang, S. Nie, Z. G. Chen, D. M. Shin, Clin. Cancer Res. 2008, 14, 1310-1316; c) S. M. Janib, A. S. Moses, J. A. MacKay, Adv. Drug Deliv. Rev. 2010, 62, 1052-1063.

[8] a) Y.-E. Koo Lee, R. Smith, R. Kopelman, Annu. Rev. Anal. Chem. 2009, 2, 57-76; b) L. M. Uusitalo, N. Hempel, Int. J. Mol. Sci. 2012, 13, 10660-10679.

[9] M. J. Ruedas-Rama, J. D. Walters, A. Orte, E. A. H. Hall, Anal. Chim. Acta 2012, 751, 1-23.

[10] a) V. M. Chauhan, G. R. Burnett, J. W. Aylott, Analyst 2011, 136, 17991801 ; b) M. Kuruppuarachchi, H. Savoie, A. Lowry, C. Alonso, R. W. Boyle, Mol. Pharm. 2011, 8, 920-931; c) S. Wang, G. Kim, Y.-E. K. Lee, H. J. Hah, M. Ethirajan, R. K. Pandey, R. Kopelman, ACS Nano 2012, 6, 6843-6851.

[11] C. Spagnul, J. Greenman, M. Wainwright, Z. Kamil, R. W. Boyle, J. Mater. Chem. B 2016, 4, 1499-1509.

[12] a) M. Brasuel, R. Kopelman, J. W. Aylott, H. Clark, H. Xu, M. Hoyer, T. J. Miller, R. Tjalkens, M. A. Philbert, Sensors Mater. 2002, 14, 309338; b) H. Xu, J. W. Aylott, R. Kopelman, Analyst 2002, 127, 1471-7; c) J. W. Aylott, Analyst 2003, 128, 309-12; d) L. B. Josefsen, J. W. Aylott, A. Beeby, P. Warburton, J. P. Boyle, C. Peers, R. W. Boyle, Photochem. Photobiol. Sci. 2010, 9, 801-811; e) F. Giuntini, F. Dumoulin, R. Daly, V. 
1
Ahsen, E. M. Scanlan, A. S. P. Lavado, J. W. Aylott, G. A. Rosser, A Beeby, R. W. Boyle, Nanoscale 2012, 4, 2034-45.

[13] K. Welser, M. D. A. Perera, J. W. Aylott, W. C. Chan, Chem. Commun 2009, 6601-6603.

Singlet Oxygen Sensor Green Product Information https://www.thermofisher.com/order/catalog/product/S36002. Visited on November 11, 2016

[14] Singlet Oxygen Sensor Green Product Information https://Www.thermofisher.com/order/catalog/product/\$36002. Visited on July $11,2016$.

a) G. Kim, Y-E. K. Lee, H. Xu, M. A. Philbert, R. Kopelman, Anal. Chem. 2010, 82, 2165-2169; b) H. Koo, M. S. Huh, J. H. Ryu, D. E. Lee, I. C. Sun, K. Choi, K. Kim, I. C. Kwon, Nano Today, 2011, 6, 204-220; c) J. P. Sumner, R. Kopelman, Analyst, 2005, 130, 528-533; d) J. P. Sumner, N. M. Westerberg, A. K. Stoddard, C. A. Fierke, R. Kopelman, Sens. Actuators, 2006, 113, 760-767.

[13] [15] K. Welser, M. D. A. Perera, J. W. Aylott, W. C. Chan, Chem. Commun. 2009, 6601-6603.
[15] [16] N. Barbero, E. Barni, C. Barolo, P. Quagliotto, G. Viscardi, L. Napione, S. Pavan, F. Bussolino, Dyes Pigments 2009, 80, 307-313.

[16] [17] a) X. Shu, V. Lev-Ram, T. J. Deerinck, Y. Qi, E. B. Ramko, M. W. Davidson, Y. Jin, M. H. Ellisman, R. Y. Tsien, PLoS Biol. 2011, 9 e1001041; b) R. Ruiz-González, A. L. Cortajarena, S. H. Mejias, M. Agut, S. Nonell, C. Flors, J. Am. Chem. Soc. 2013, 135, 9564-9567; c) F. M. Pimenta, R. L. Jensen, T. Breitenbach, M. Etzerodt, P. R. Ogilby, Photochem. Photobiol. 2013, 89, 1116-26.

[17] [18] a) R. Ruiz-González, J. H. White, M. Agut, S. Nonell, C. Flors Photochem. Photobiol. Sci. 2012, 11, 1411-1413; b) R. Ruiz-González, J. H. White, A. L. Cortajarena, M. Agut, S. Nonell, C. Flors, Proc. SPIE, 2013, 8596, 859609.

[18] [19] K. Berg, A. Weyergang, M. Vikdal, O.-J. Norum, M. Berstad, P. Selbo, Photodiagn. Photodyn. Ther. 2011, 8, 155-156.

[20] M. Wagner, P. Weber, T. Bruns, W. S. L. Strauss, R. Wittig, H. Schneckenburger, Int. J. Mol. Sci. 2010, 11, 956-966. 
5

Entry for the Table of Contents (Please choose one layout)

\section{COMMUNICATION}

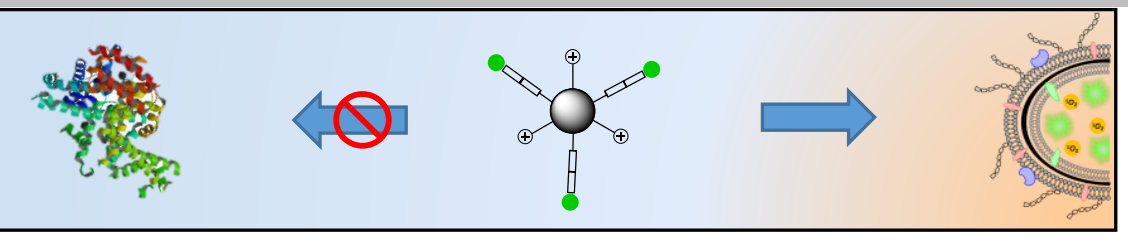

When bound to nanoparticles, the fluorescent probe Singlet Oxygen Sensor Green ${ }^{\circledR}$ escapes protein sequestration, readily undergoes cell uptake, and efficiently reports on intracellular variations of singlet oxygen levels.
Rubén Ruiz-González, Roger BresolíObach, Òscar Gulías, Montserrat Agut, Huguette Savoie, Ross W. Boyle, * Santi Nonell* and Francesca Giuntini*

Page No. - Page No.

NanoSOSG: a Nanostructured Fluorescent Probe for Intracellular Singlet Oxygen Detection 